\title{
Three Dimensional Graphene Transistor for Ultra-sensitive pH Sensing Directly in Biological Media
}

\author{
Shideh Kabiri Ameri, Pramod K. Singh, Sameer Sonkusale* \\ Nanolab, Department of Electrical and Computer Engineering, Tufts University \\ 161 College Ave, Medford, MA 02155, USA \\ Email: sameer@ece.tufts.edu
}

\begin{abstract}
In this work, $\mathrm{pH}$ sensing directly in biological media using three dimensional liquid gated graphene transistors is presented. The sensor is made of suspended network of graphene coated all around with thin layer of hafnium oxide $\left(\mathrm{HfO}_{2}\right)$, showing high sensitivity and sensing beyond the Debye-screening limit. The performance of the $\mathrm{pH}$ sensor is validated by measuring the $\mathrm{pH}$ of isotonic buffered solution Dulbecco's phosphate buffered saline (DPBS), and of blood serum derived from a Sprague-Dawley rat. The $\mathrm{pH}$ sensor shows high sensitivity of $71 \pm 7 \mathrm{mV} / \mathrm{pH}$ even in high ionic strength media with molarities as high as $289 \pm 1 \mathrm{mM}$. High sensitivity of this device is owing to suspension of three dimensional graphene in electrolyte which provides all around liquid gating of graphene, leading to the higher electrostatic coupling efficiency of electrolyte to the channel and higher gating control of transistor channel by ions in the electrolyte. Coating graphene with hafnium oxide film $\left(\mathrm{HfO}_{2}\right)$ provides binding sites for hydrogen ions increasing the overall sensitivity and sensing beyond the Debye-screening limit. The 3D graphene transistor offers the possibility of real-time $\mathrm{pH}$ measurement in biological media without need of desaltation or sample preparation.
\end{abstract}

\section{Introduction}

Graphene has attracted a lot of attention in recent years for various electronic ${ }^{1,2}$, sensing and bioelectronics $^{3,4,5,6,7}$ applications due to its high carrier mobility, high carrier concentration, biocompatibility, atomic thickness, electrochemical stability and mechanical reliability ${ }^{8}$. Many different biomolecules have been sensed using liquid-gated graphene devices where a solid-state dielectric is 
replaced by electrolytic double layer capacitance formed at the liquid-graphene interface ${ }^{9,10}$. Such configuration enables direct sensing of the liquid environment. Due to atomic thickness of graphene, its carrier density is very sensitive to the charge species in the liquid environment which enables chargedetection based mechanism of sensing for charged molecules and ions ${ }^{10,11,12}$. One of the charge detection based sensing using liquid gated graphene FETs, reported in literature is $\mathrm{pH}$ sensing, however reported $\mathrm{pH}$ sensing performance has not been consistent and sensitivities vary widely from 17 to $99 \mathrm{mV} / \mathrm{pH}$ $10,13,14,15,16$. Recently, it has been shown that a perfect defect-free graphene transistor is in fact not sensitive to the ions in the environment and the $\mathrm{pH}$ sensing may be mediated by existence of defects and dangling bonds on graphene surface and edges and leakage current through bare source and drain gold contacts ${ }^{16}$. Another problem associated with charge-detection based sensing of $\mathrm{pH}$, especially for biological applications is the limited dynamic range of sensitivity due to charge screening in high ionic strength of biological medium. Presence of electrical double layer at graphene/electrolyte interface results in exponential drop of potential away from the graphene/electrolyte interface. This effect is captured by the characteristic length known as Debye length beyond which the electrostatic effect of ions on the graphene channel is minimal. Debye length is defined as ${ }^{10}$

$$
\lambda_{D}=\sqrt{\frac{\varepsilon k T}{e^{2} \sum_{i} Z_{i}^{2} n_{i 0}}}
$$

Where $\varepsilon$ is permittivity, $K$ is the Boltzmann's constant, $T$ is temperature, $e$ is electron charge and $Z_{i}$ is charge of the species $i$ and $n_{i 0}$ is the concentration (number per volume) of the species $i$ under equilibrium neutrality. At a constant temperature, Debye length just depends on the permittivity and ionic strength of the electrolyte. As an example, in a $100 \mathrm{mM}$ aqueous electrolyte at room temperature, the Debye length is only about $1.3 \mathrm{~nm}$, beyond which the charge carriers in the bulk does not affect channel potential. In the most biological medium, concentration is higher than $100 \mathrm{mM}$. The osmolality of extracellular fluids 
and human blood serum is between 277 to $305 \mathrm{mOsmol} / \mathrm{Kg}$, which correspond to 270 to $297.4 \mathrm{mM}^{17}$; and Debye length in such high concentration medium will be even smaller. Therefore to be able to perform charge-detection based sensing of charged molecules and ions in biological samples like as blood serum, urine and extracellular fluids, one needs to perform desaltation of sample to reduce the overall ionic strength $^{18}$. Sensing $\mathrm{pH}$ using graphene based $\mathrm{pH}$ sensors has not been reported in medium with concentration more than $100 \mathrm{mM}$ due to such charge screening effect ${ }^{13-16}$. This limits the practical use of current graphene based $\mathrm{pH}$ sensors in real time monitoring of $\mathrm{pH}$ and other ions directly in the blood and biological fluids, which can be extremely useful especially in emergency medical care. For example, it is known that if arterial blood $\mathrm{pH}$ falls below 7.37 (normal range is 7.37 to 7.42 ), acidosis occurs ${ }^{19}$. Severe acidosis can lead to shock and death ${ }^{20}$. Therefore real time monitoring of $\mathrm{pH}$ of blood serum can be very valuable.

It has been shown that by adding oxide on graphene, the $\mathrm{OH}$ groups at the surface of oxide in contact with electrolyte can be protonated or deprotonated based on the $\mathrm{pH}$ of the solution and oxide surface composition $^{21,22,23,24}$. In this case, the sensitivity of the device is not dependent on the electrolyte concentration $^{25}$. Sensing $\mathrm{pH}$ using graphene-based liquid-gated field effect transistor (FET) with aluminum oxide dielectric acting as an ion sensitive layer has been reported ${ }^{16}$. It is shown that by growing less than $2 \mathrm{~nm}$ of $\mathrm{Al}_{2} \mathrm{O}_{3}$ using atomic layer deposition (ALD) at low temperature, the sensitivity of the sensor increases from $6 \mathrm{mV} / \mathrm{pH}$ to $17 \pm 2 \mathrm{mV} / \mathrm{pH}^{16}$. In another work, the sensitivity of 40 to $50 \mathrm{mV} / \mathrm{pH}$ was achieved by adding high quality of aluminum oxide on the graphene ${ }^{22}$.The low sensitivity of earlier work is attributed to the poor quality of the oxide grown on graphene, due to low temperature growth process and low thickness of oxide (less than $2 \mathrm{~nm}$ ). The sensitivity of such devices also depends on the type of oxide used as a sensing surface. It has been shown that using hafnium oxide, $\mathrm{HfO}_{2}$, provides higher $\mathrm{pH}$ sensitivity of FET up to $59 \mathrm{mV} / \mathrm{pH}^{26}$.

The pH sensitivity of conventional FETs and ISFETs is theoretically obtained using Nernst equation. Based on this equation the maximum sensitivity such devices to $\mathrm{pH}$ is limited to $59 \mathrm{mV} / \mathrm{pH}$, which comes 
from the relation between changes of electrostatic potential at the insulator-electrolyte interface, $\varphi_{0}$, with change of bulk electrolyte $\mathrm{pH}, \mathrm{pH}_{B}$, given by ${ }^{25}$

$$
\begin{aligned}
& \frac{\partial \varphi_{0}}{\partial p H_{B}}=-2.3 \frac{k T}{e} \alpha \\
& \alpha=\frac{1}{\left(\frac{2.3 k T C_{\text {dif }}}{e^{2} \beta_{\text {int }}}\right)+1}
\end{aligned}
$$

where $\alpha$ is dimensionless sensitivity parameter, $C_{\mathrm{dif}}$ is differential capacitance, $\beta_{\mathrm{int}}$ is intrinsic buffer capacity, $k$ is Boltzmann constant, $T$ is temperature, and $e$ is electron charge. The sensitivity parameter is the function of differential capacitance and buffer capacity, which depends on the density of sensing sites on the surface and the ions in the sensing liquid ${ }^{25}$. The maximum value of $\mathrm{pH}$ sensitivity given by equation 2 is $59 \mathrm{mV} / \mathrm{pH}$ at room temperature, known as Nernst limit. But one should notice that to derive Nernst equation, the oxide/electrolyte interface is modeled using either $\mathrm{Hg} /$ electrolyte or AgI/electrolyte interface so this equation is not valid at the oxide/electrolyte surface where variation of charge density with electrostatic potential, $\varphi_{0}$, is very different than $\mathrm{Hg} /$ electrolyte and $\mathrm{AgI} /$ electrolyte interfaces and in most of the oxides the surface charge density and differential capacities are higher than those in $\mathrm{Hg}$ and $\operatorname{AgI}^{25,26,27,28}$. It has been shown that sensitivity of the field effect transistors can be tuned by using

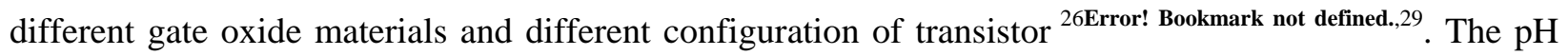
sensitivity reported in dual gated FETs is considerably higher than Nernst limit ${ }^{30,31}$. The second gate in dual gated FETs enables compensation of the change in current induced by the first gate in response to $\mathrm{pH}$ which results in an effective amplification of the sensitivity. The super sensitivity of porous gate ISFET has also been reported where the effect was attributed to the $3 \mathrm{D}$ structure of the gate, results in the effective increase in the adsorption surface area and increased buffer capacity ${ }^{32}$.

In this work, a three-dimensional suspended graphene is used as the channel of a liquid gated transistor. Graphene is coated with $20 \mathrm{~nm}$ hafnium oxide using atomic layer deposition (ALD) which acts as a pH sensing surface. The three-dimensional nature of this macro porous oxide-coated graphene foam results in 
a considerably larger sensing surface area. Moreover, the suspended structure of the liquid gated FET enables ions to accumulate all around on exposed surfaces resulting in significant improvement in liquid gate/channel coupling efficiency ${ }^{33}$. These effects have resulted in the proposed graphene transistor device to demonstrate a $\mathrm{pH}$ sensitivity of $71 \pm 7 \mathrm{mV} / \mathrm{pH}$, with the advantages of biocompatibility, potential flexibility and sensitivity in biological media with high ionic concentration.

\section{Methods}

The fabrication process flow of $\mathrm{pH}$ sensor is shown in figure 1. Process begins with placing CVD grown graphene (Graphene Supermarket Inc. USA) in ferric chloride solution $\left(\mathrm{FeCl}_{3}, 0.06 \mathrm{M}\right)$ to etch away copper and then rinsing it with sufficient amount of deionized water (DI water) to remove the residue left over from the etching process. Titanium and gold were deposited through shadow mask using DC magnetron sputtering (NSC 3000) with the thickness of $30 \mathrm{~nm}$ and $220 \mathrm{~nm}$ respectively to form source and drain contacts. The length and width of transistor were $600 \mu \mathrm{m}$ and $5 \mathrm{~mm}$, respectively. In the next step, $20 \mathrm{~nm}$ of $\mathrm{HfO}_{2}$ was deposited using atomic layer deposition (Savannah ALD, Cambridge NanoTech) all over the device. Then an additional thin layer of PDMS (Polydimethylsiloxane) was added as a

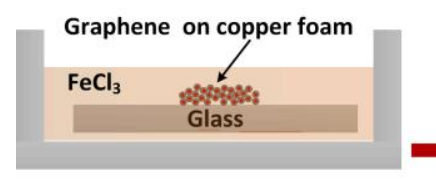

(a)

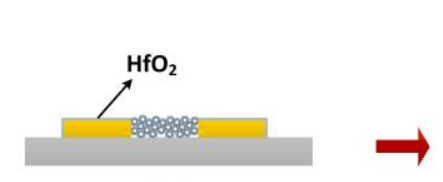

(e)

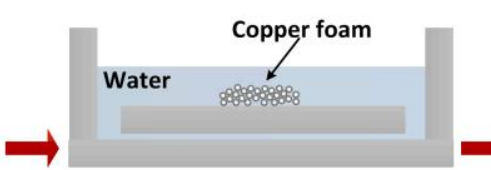

(b)

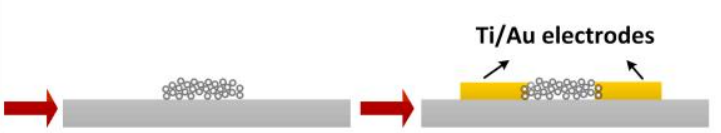

(c)

(d)

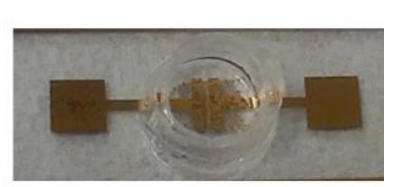

(h)

Figure 1. Cross sectional view of the sensor fabrication process. (a) CVD grown graphene on copper foam is placed in $\mathrm{FeCl}_{3}$. (b) After etching away copper, graphene is rinsed with DI water. (c) Graphene foam is dried. (d) $\mathrm{Ti} / \mathrm{Au}$ is deposited through shadow mask to form source and drain connections. (e) $\mathrm{HfO}_{2}$ is grown on the device. (f) Contacts area close to the channel is covered with PDMS protective layer. (g) PDMS well is fabricated and attached to the device. (h) Photo of the fabricated device with PDMS well.

protective layer to prevent any direct physical contact between gold (source and drain contacts) and electrolyte thus minimizing leakage current. A well is made around the channel using PDMS and bonded 
to the device to prevent spreading of the electrolyte on the device. Dulbecco phosphate buffered saline (DPBS) (calcium chloride, $\mathrm{CaCl}_{2}, 1 \mathrm{gL}^{-1}$, magnesium chloride, $\mathrm{MgCl}_{2} \cdot 6 \mathrm{H}_{2} \mathrm{O}, 1 \mathrm{gL}^{-1}$, potassium chloride, $\mathrm{KCl}, 2 \mathrm{gL}^{-1}$, potassium phosphate monobasic, $\mathrm{KH}_{2} \mathrm{PO}_{4}, 2 \mathrm{gL}^{-1}$, sodium chloride, $\mathrm{NaCl}, 80 \mathrm{gL}^{-1}$, sodium phosphate dibasic, $\mathrm{Na}_{2} \mathrm{HPO}_{4} \cdot 7 \mathrm{H}_{2} \mathrm{O}, 21.6 \mathrm{gL}^{-1}$, water) ${ }^{34}$ and blood serum of a male sprague-dawley rat were used to test the functionality of the $\mathrm{pH}$ sensor. Different DPBS $(150 \mathrm{mM})$ based $\mathrm{pH}$ solutions with $\mathrm{pH}$ range from 3 to 9 were made by adding sodium hydroxide $(\mathrm{NaOH})$ and Hydrochloric acid $(\mathrm{HCl})$. The rat blood serum at four different $\mathrm{pH}$ values between 6.4 and 7.7 was used for testing the functionality of sensor directly in biological medium. The choice of the $\mathrm{pH}$ matches the known critical range of blood $\mathrm{pH}$ for diagnosis of acidosis. The initial $\mathrm{pH}$ of the serum extracted from the rat was measured to be 7.7. To provide blood serum with different $\mathrm{pH}$, the blood was first extracted from a male Sprague-Dawley rat and added to a tube containing polymer gel for separating the serum. After separation the serum, it was divided in four parts. To simulate the blood acidosis, the $\mathrm{pH}$ of serum was adjusted at four different $\mathrm{pH}$ values from 6.4 to 7.7 by spiking it with appropriate amount of diluted hydrochloric acid, $0.03 \mathrm{M}$, through a nafion membrane. Since nafion is permeable only to the protons in the solution, we could simulate acidosis of blood by spiking the blood with hydrogen ions in $\mathrm{HCl}$. The serum was stirred using a magnetic stirrer continuously to distribute the hydrogen ions that penetrated through the membrane evenly in the serum; the process was monitored using a $\mathrm{pH}$ meter. Once the $\mathrm{pH}$ of serum reached the desired value, it was used as a test sample.

\section{Results and discussions}



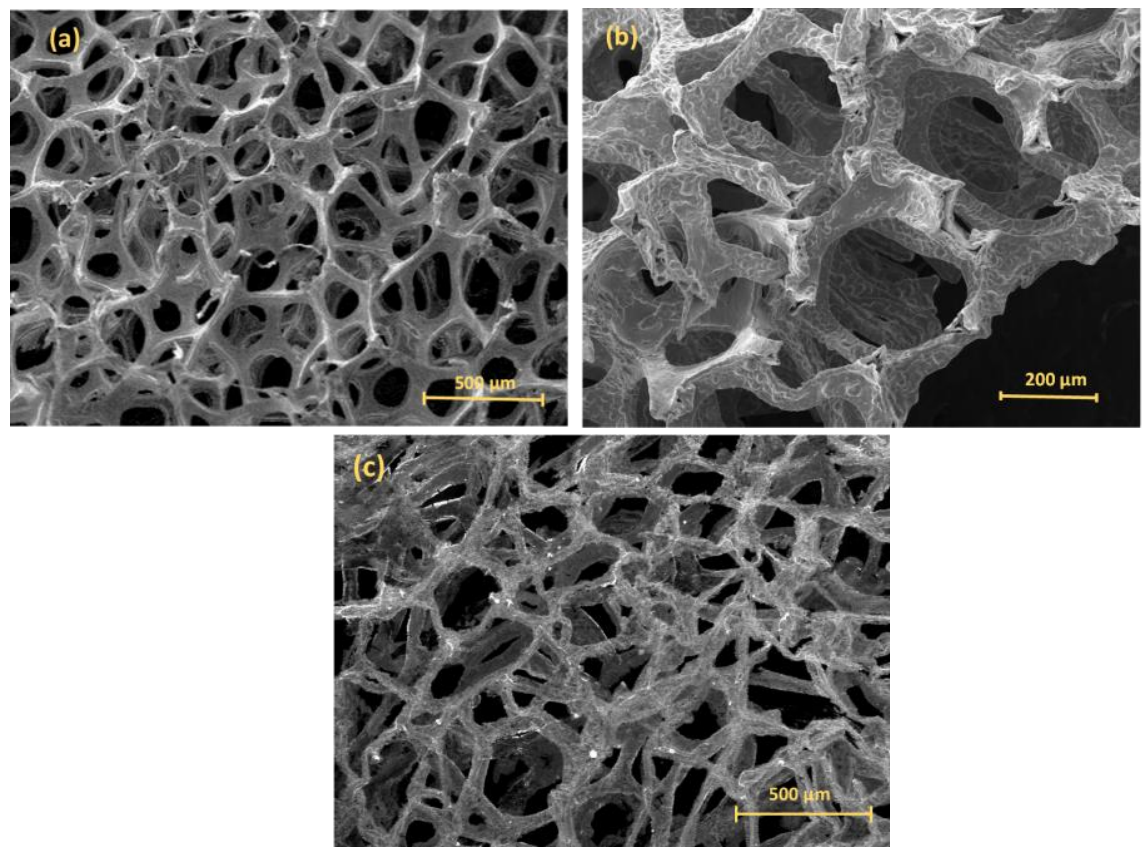

Figure 2. SEM image of the graphene foam; (a) few layers graphene grown on copper foam; (b) graphene foam coated with $20 \mathrm{~nm}$ of $\mathrm{HfO}_{2}$; (c) the SEM image of graphene foam after etching the copper.

The channel of the presented transistor is made of CVD grown graphene on the copper foam. The SEM image of graphene grown on copper foam is shown in figure 2. After etching away the copper, graphene collapses but still maintains it's initial three dimensional structure (see figure $2 \mathrm{c})^{35}$. Our previous study indicates that the graphene foam, is made of few layers of graphene ${ }^{35}$. As mentioned earlier, hafnium oxide as a sensing surface with the thickness of $20 \mathrm{~nm}$ was grown on the graphene after forming contacts. Due to the sp2 crystalline structure of graphene, hydrophobicity and lack of dangling bonds, growth of $\mathrm{HfO}_{2}$ on graphene using $\mathrm{ALD}$ is challenging. Basically, the growth of $\mathrm{HfO}_{2}$ on graphene starts at defects or boundaries of bilayer graphene islands, which usually form during the growth of monolayer graphene on copper and act as nucleation sites. It continues with formation of islands of oxide, grow in size and at a sufficient thickness merge with other islands and form a continuous layer ${ }^{36}$. To investigate the quality and continuity of the $\mathrm{HfO}_{2}$ grown on graphene, X-ray photoelectron spectroscopy (XPS) of $\mathrm{HfO}_{2}$ with the thickness of $20 \mathrm{~nm}$ grown on the graphene before etching away copper was performed at several random spots. The X-ray spot size was set to be $200 \mu \mathrm{m}$. Considering the fact that, the XPS only provides surface information and X-ray in XPS penetrates only 
10 to $20 \mathrm{~nm}$ in the investigated layer and also that the thickness of the grown $\mathrm{HfO}_{2}$ on graphene was 20 $\mathrm{nm}$, one can correlate absence of copper peaks to deduce a presence of continuous layer of oxide at the top of the graphene. Figure 3(a) shows the XPS results. As seen, all of the related peaks of $\mathrm{HfO}_{2}$ exist in XPS spectrum. The C1s peak reveals the existence of carbon in the oxide layer, attributed to the organic residue from precursor decomposition

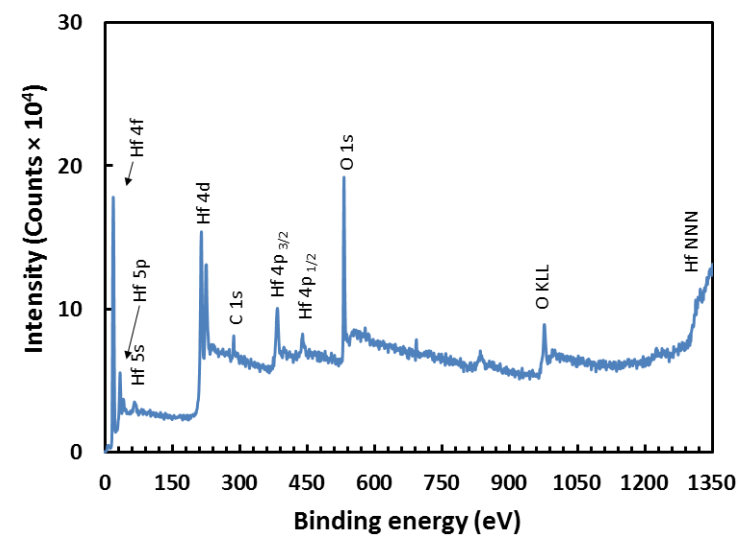

(a)

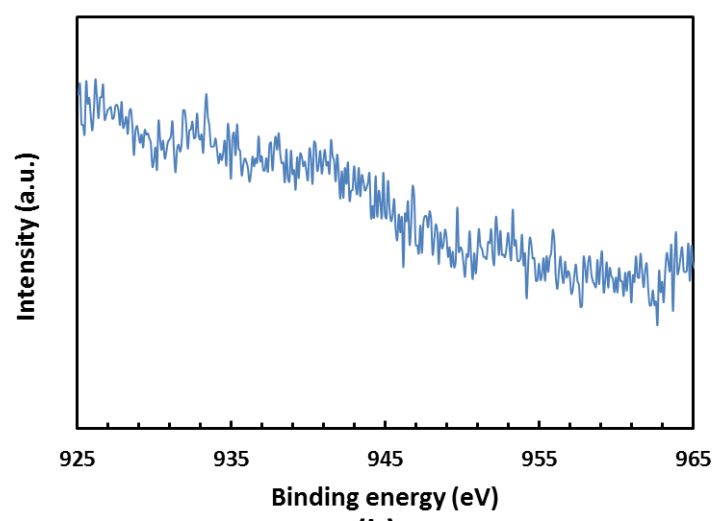

(b)

Figure 3. XPS of hafnium oxide on graphene foam; (a) The XPS of $\mathrm{HfO}_{2}$ with $20 \mathrm{~nm}$ thickness on the graphene before etching away the copper foam; (b) high resolution XPS spectra in copper binding energy range.

during growth ${ }^{37}$. Figure 3(b) shows high-resolution XPS spectra in the copper binding energy range, which shows absence of the copper peak, confirming the continuity of the $\mathrm{HfO}_{2}$ films on the graphene. Sensing was performed in ambient environment at atmospheric pressure and room temperature. The leakage current was measured to be at least three orders of magnitude lower than drain to source current. Figure 4(a) shows the transfer characteristics of the transistor gated with different DPBS based $\mathrm{pH}$ solutions with the $\mathrm{pH}$ values between 3 to 9 . DPBS is a typical isotonic solution with molarity of about $150 \mathrm{mM}$, widely used for biological applications. With increasing $\mathrm{pH}$ of solution the Dirac point shifts towards more positive voltages. This behavior can be explained using site binding model ${ }^{24}$, which depending on the $\mathrm{pH}$ of the electrolyte, oxide surface composition and hydrophilicity, the $\mathrm{OH}$ groups on the surface of oxide can protonate to form $\mathrm{OH}_{2}{ }^{+}$, remain neutral, or deprotonate to form $\mathrm{O}^{-16}$. When $\mathrm{pH}$ of solution is acidic, $\mathrm{OH}$ groups protonate and as a result Dirac point shifts toward more negative voltages 
and when $\mathrm{pH}$ of solution is basic, $\mathrm{OH}$ groups deprotonate and the Dirac point shifts toward more positive voltages. Figure 4(b) shows that the shift in Dirac point voltage of the device with $\mathrm{pH}$ change is linear. The error bars represent the variation of voltage shift for four devices fabricated and tested under the same conditions. The sensitivity of $71 \pm 7 \mathrm{mV} / \mathrm{pH}$ was deduced for these sensors, which is greater than the Nernst limit. As explained earlier, the higher sensitivity is attributed to the larger sensing surface area 

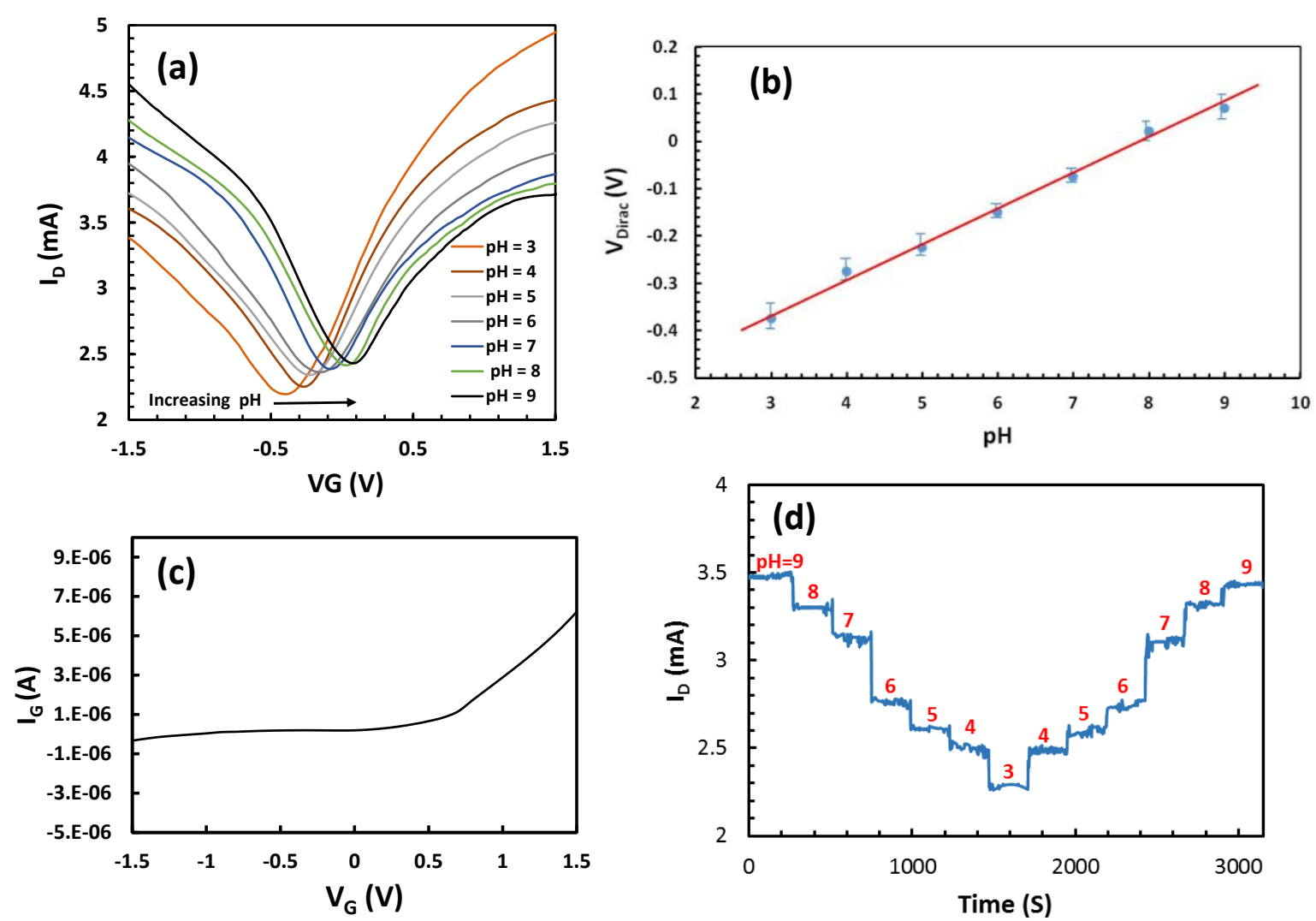

Figure 4. Sensing results and sensor characterization; (a) transfer characteristics of transistor for different $\mathrm{pH}$ of DPBS; (b) shift of gate voltage at Dirac point at different $\mathrm{pH}$ solutions; (c) Gate leakage current of transistor at $\mathrm{V}_{\mathrm{d}}=0.5 \mathrm{~V}, \mathrm{pH}=5$; (d) the real time monitoring of the $\mathrm{pH}$ with changing of the $\mathrm{pH}$ from 9 to 3 and then from 3 to 9.

and the high density of binding sites on $\mathrm{HfO}_{2}$. The resultant high charge accumulation on surface and the all-around liquid gating of the transistor results in higher gate channel coupling efficiency which causes large Dirac shift in graphene transistor with small changes in $\mathrm{pH}$. It should be noted here that the role of electrolytic double layer capacitance and the oxide capacitance in the Id-Vg shift is not as prominent as the quantum capacitance, which is in series with the other two; the increased sensitivity is primarily due to increased buffer capacity from large sensing surface area. Proposed device can perform sensing in higher ionic strength electrolyte background, which is typically known to be challenging for ISFETs ${ }^{13,14,22,38}$. Coating 3D graphene with $\mathrm{HfO}_{2}$ as a sensing surface provides site binding for hydrogen 

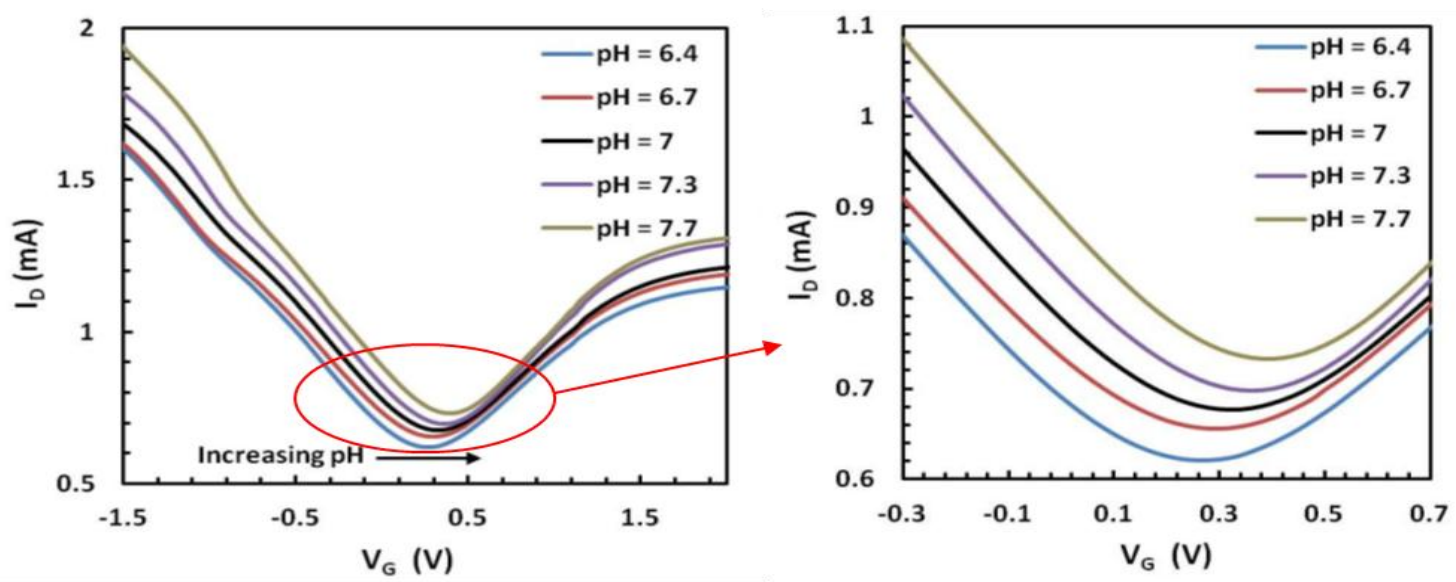

Figure 5. Transfer characteristics of the transistor for blood serum at different $\mathrm{pH}$ values.

ions in electrolyte and contribute to the sensing beyond Debye length. In fact, binding ions to the surface of $\mathrm{HfO}_{2}$ converts charge accumulation to the conductivity change in graphene. Also all around coating of the graphene with $\mathrm{HfO}_{2}$ as a high $\mathrm{K}$ dielectric as well as electrolyte, provides higher control of the gate and consequently higher sensitivity of the device. In this device gate leakage current was measured to be three orders of magnitude lower than drain current as shown in figure 4(c). The real time monitoring of the $\mathrm{pH}$ was demonstrated and result is shown in figure 4(d). In this experiment, gate to source voltage was fixed at $-0.5 \mathrm{~V}$ and drain to source voltage was set at $0.5 \mathrm{~V}$. The solutions with different $\mathrm{pH}$ from 9 to 3 and then 3 to 9 were added at the top of the channel of transistor at 240-second intervals. The change in the drain current was then recorded for different $\mathrm{pH}$ solutions as a function of time. The result is consistent with the change in transfer characteristics of the transistor in the hole conduction regime (at $\mathrm{V}_{\mathrm{G}}$ $=-0.5 \mathrm{~V}$ ) for different $\mathrm{pH}$ of electrolyte. Results show good reversibility for sensing $\mathrm{pH}$ between 3 and 9 using the presented device.

To investigate the functionality of the sensor in body fluids, blood serum of a rat $(296 \pm 1$ $\mathrm{mOsm} / \mathrm{kg}$ ) at four different $\mathrm{pH}$ values from 6.4 to 7.7 was used. Rat blood serum with the $\mathrm{pH}$ of 7 first was added in the well. After measurement the serum was removed and measurement was continued with adding serum with $\mathrm{pH}$ of 7.3 and 7.7. Then the device was washed with deionized water and tested using serum with $\mathrm{pH}$ of 6.7 and 6.4 respectively. Results of sensing $\mathrm{pH}$ of the serum are shown in figure 
5. As seen, the Dirac point shifts clearly toward more positive voltages with increasing $\mathrm{pH}$ from 6.4 to 7.7. The sensitivity of the device was not affected by the high ionic strength of the background electrolyte of around $289 \mathrm{mM}$ in the rat blood serum compared to $150 \mathrm{mM}$ in DPBS, indicating the ability of the sensor to work directly in biological medium without the need for desaltation or sample preparation. One should notice that the background charged molecules in the blood plasma would also be adsorbed on to $\mathrm{HfO}_{2}$ layer and thus influence the $\mathrm{pH}$ measurement. The sensor should therefore be characterized for all the possible variation in the blood background to parameterize this dependence. This is beyond the scope of this paper and could be focus of future work.

\section{Conclusions}

In conclusion, a highly sensitive $\mathrm{pH}$ sensor is demonstrated based on 3D liquid gated graphene transistor. The channel of this device consists of suspended three dimensional suspended graphene network covered all around with $\mathrm{HfO}_{2}$ layer as a sensing surface. Device shows the high pH sensitivity of $71 \pm 7 \mathrm{mV} / \mathrm{pH}$ exceeding the Nernst limit even in high ionic strength media. The superior performance of the device is attributed to the suspended configuration and the high surface area of the three dimensional graphene which provides all around electrostatic gate control. This results in enhancement of coupling efficiency between ions in the electrolyte and the graphene channel. High surface area of $\mathrm{HfO}_{2}$, coated all around graphene provides binding sites for hydrogen ions and enables high sensitivity and sensing beyond the Debye-screening limit in high ionic strength media. This device can perform reliably in the high ionic strength biological media such as DPBS and blood serum and has a potential to be used for the real time monitoring of $\mathrm{pH}$ directly in such biological environment, with applications in medicine and life sciences. One application is that of monitoring blood $\mathrm{pH}$ in emergency medical care for real-time monitoring of acidosis in patients, and another application is that monitoring in-vitro $\mathrm{pH}$ of cells and tissue in tissue engineering and drug screening applications.

\section{Acknowledgment}


We would like to thank Professor Qiaobing Xu and Kyle Alberti for supplying us with rat blood

for measurements, and for the helpful discussions and suggestions. The authors also acknowledge the partial support of National Science Foundation (NSF) grant NSF ECCS-0955024.

\section{References}

(1) Wu, Y.; Jenkins, K. A.; Garcia, A. V.; Farmer, D. B.; Zhu, Y. et al, Nano Lett. 2012, 12, 3062-3067.

(2) Wu, Y.; Lin, Y. M.; Bol, A. A.; Jenkins, K. A.; Xia, F. et al. Nature 2011, 472, 74-78.

(3) Ameri, S. K.; Singh, P. K.; Sonkusale, S. Biosensors and Bioelectronics 2014, 61, 625-630.

(4) Hess, L. H.; Jansen, M.; Maybeck, V.; Hauf, M. V.; Seifert, M.; et al. Adv. Mater. 2011, 23, 5045-5049.

(5) Shao, Y.; Wang, J.; Wu, H.; Liu, J.; Aksay, I. A.; et al. Electroanalysis 2010, 22, 1027 - 1036.

(6) Sasidharan, A.; Panchakarla, L. S.; Sadanandan, A. R.; Ashokan, A.; Chandran, P.; et al. Small 2012, 8, 12511263.

(7) Yang, K.; Zhang, S.; Zhang, G.; Sun, X.; Lee, S. T.; et al. Nano Lett. 2010, 10, 3318-3323.

(8) Geim, A. K. Science 2009, 324, 1530-4.

(9) Lu, C. H.; Yang, H. H.; Zhu, C. L.; Chen, X.; Chen, G. N. Angew. Chem. Int. Ed. 2009, 48, $4785-4787$.

(10) Kulkarni, G. S.; Zhong, Z. Nano Lett. 2012, 12, 719-723.

(11) Yuan, W.; Shi, G. J. Mater. Chem. A 2013, 1, 10078-10091.

(12) Ko, G.; Kim, H. Y.; Ahn, J.; Park, Y. M.; Lee, K. Y.; Kim, J. Applied Physics 2010, 10, 1002-1004.

(13) Giacchetti, B. M.; Hsu, A.; Wang, H.; Vinciguerra, V.; Pappalardo, F.; et al. J. Appl. Phys. 2013,114, 084505.

(14) Tan, X.; Chuang, H. J.; Lin, M. W.; Zhou, Z.; Cheng, M. M. C. J. Phys. Chem. C 2013, 117, 27155-27160.

(15) Ang, P. K.; Chen, W.; Wee, A. T. S.; Loh, K. P. J. Am. Chem. Soc. 2008, 130, 14392-14393.

(16) Fu, W.; Nef, C.; Knopfmacher, O.; Tarasov, A.; Weiss, M. Nano Lett. 2011, 11, 3597-3600.

(17) Waymouth, C. In vitro 1970, 6, 109-127.

(18) Zheng, G. F.; Patolsky, F.; Cui, Y.; Wang, W. U.; Lieber, C. M. Nat. Biotechnol. 2005, 23, 1294-1301.

(19) Oliva, P. B. The American journal of medicine 1970, 48, 209-225.

(20) Hermaxsels, L.; Osnes, J. B. J. Applied Physiology 1972, 32, 304-308.

(21) Chen, S.; Bomer, J. G.; van der Wiel, W. G.; Carlen, E. T.; van den Berg, A. ACS Nano 2009, 3, 3485-3492.

(22) Wang, B.; Liddell, K. L.; Wang, J.; Koger, B.; Keating, C. D.; Zhu, J. Nano Research 2014, 7, 1263-1270.

(23) Zafar, S.; D’Emic, C.; Afzali, A.; Fletcher, B.; Zhu, Y.; Ning, T. Nanotechnology 2011, 22, 405501.

(24) Yatess, D.; Vine, A.; Healy, T. W. J. Chem. Soc. Faraday Trans. 1 1974, 70, 1807-1818.

(25) van Hal, R. E. G.; Eijkel, J. C. T.; Bergveld, P. Sensors and Actuators B 1995, 24-25, 201-205.

(26) Berube, Y. G.; de Bruyn, P. L. J. Colloid Interface Sci. 1968, 27, 305-318.

(27) Levine S.; Smith, A. L. Disc. Faraday Soc. 1971, 52, 290-301.

(28) Yates, D. E.; Levine, S.; Healy, T. W. J. Chem. Soc. Faraday Trans. 1974, 70, 1807-1818.

(29) Lue , C. E.; Yu , T. C.; Yang, C. M.; Pijanowska, D. G.; La, C. S. Sensors 2011, 11, 4562-4571.

(30) Knopfmacher, O.; Tarasov, A., Fu, W.; Wipf, M.; Niesen, B.; Calame, M.; and Schönenberger, C. Nano Lett. 2010, 10, 2268-2274.

(31) Spijkman, M.; Smits, E. C. P.; Cillessen, J. F. M.; Biscarini, F.; Blom, P. W. M.; de Leeuw, D. M. Appl. Phys. Lett. 2011, 98, 043502.

(32) Zehfroosh, N.; Shahmohammadi, M.; Mohajerzadeh, S. IEEE Electron Device Lett. 2010, 31, 1056-1058.

(33) Wang, F.; Stepanov, P.; Gray, M.; Lau, C. N.; Itkis, M. E.; Haddon, R. C. Nano Lett. 2015, 15, 5284-5288.

(34) Dulbecco, R.; Vogt, M. JEM 1954, 99, 167-182.

(35) Ameri, S. K.; Singh, P. K.; Sonkusale, S. R. Carbon 2014, 79, 572-577.

(36) Lupina, G.; Lukosius, M.; Kitzmann, J.; Dabrowski, J.; Wolff, A.; et al. Appl. Phys. Lett. 2013,103, 183116.

(37) Barreca, D.; Milanov, A.; Fischer, R. A.; Devi, A.; Tondello, E. Surface Science Spectra 2007, 14, 34-40.

(38) Cheng, Z.; Li, Q.; Li, Z.; Zhou, Q.; Fang, Y. Nano Lett. 2010, 10, 1864-1868. 


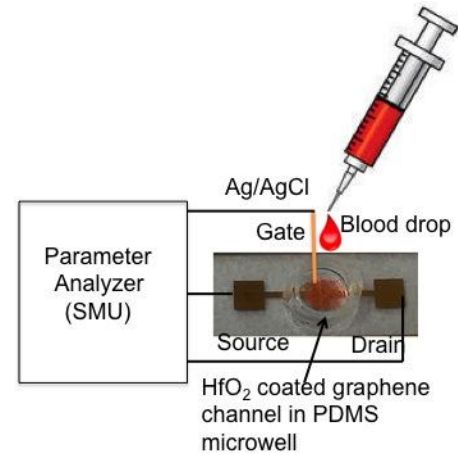

(a)

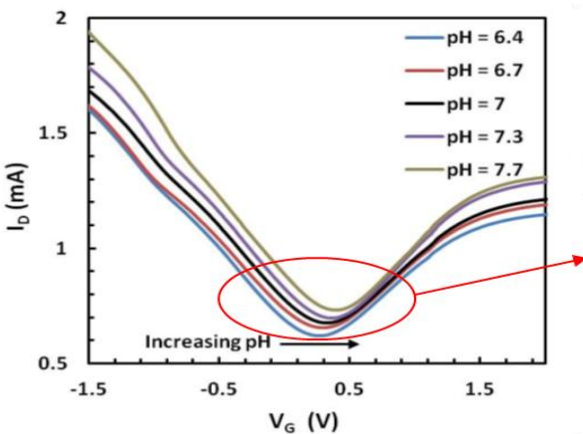

(b)

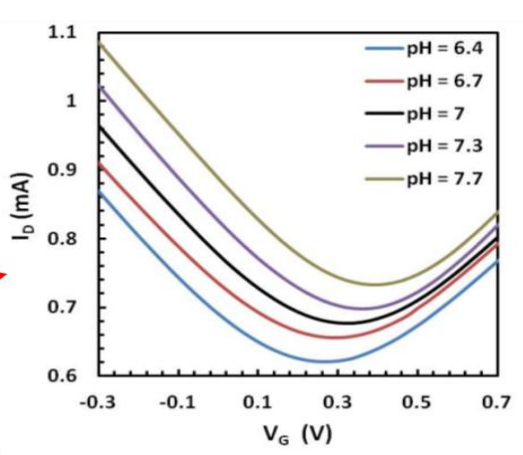

(c)

(a) Test setup - Direct rat blood serum $\mathrm{pH}$ measurements (b) Measured transfer characteristics of the transistor for blood serum at different $\mathrm{pH}$ values, and (c) Zoomed in version around direct point 